\title{
Comparación de dos métodos de liberación del berrendo, Antilocapra americana (Artiodactyla: Antilocapridae) en Coahuila, México
}

\author{
Hugo Sotelo Gallardo ${ }^{1}$, Armando J. Contreras Balderas ${ }^{2}$ \& Alejandro Espinosa Treviño ${ }^{1}$ \\ 1. CEMEX, Independencia 901-A Oriente, Colonia Cementos. Monterrey, Nuevo León, México. Apartado Postal 64520; \\ ugosotelo@hotmail.com, alejandro.espinosa@cemex.com \\ 2. Laboratorio de Ornitología, Facultad de Ciencias Biológicas, Universidad Autónoma de Nuevo León. Apartado Postal \\ 425. San Nicolás de los Garza, Nuevo León, México. 66450; ornitologia@gmail.com
}

Recibido 18-VIII-2016. Corregido 27-IV-2017. Aceptado 31-V-2017.

\begin{abstract}
Comparison of two release methods for Antilocapra americana (Artiodactyla: Antilocapridae) in Coahuila, México. The reintroduction of missing mammal species in former habitats has recently been of high interest. In Mexico, there have been several attempts to reintroduce Antilocapra since 1967, but until now none of the trials has been successful. Nowadays, different releasing methods have been practiced for mammal species, including soft and hard release ones. The aim of this study was to provide new information, and to evaluate the success of a recent release. The study was based on the monitoring of 100 individuals (70 females, 30 males) captured in New Mexico, USA, to be released in Maderas del Carmen, Coahuila, Mexico, in two different groups. The first group (fast release) of 45 specimens (20 females, 25 males) was released in the valley at the beginning of March 2009. The second one (soft release), with 55 individuals ( 50 female, 5 male), was released in March 2010. For both groups, we compared the mortality rate between dispersal and soft-release vs. hard-release methods. Our results showed that the release with adaptation gave highly significant results $\left(\chi^{2}=\right.$ $2, \alpha=0.05, \mathrm{p}=0.0001)$ ). The comparison of mortality and dispersion among both methods was highly contrasting: with soft-release we obtained $4 \%$ and $13 \%$ of success, against $23 \%$ and $46 \%$, respectively. Considering these results, we recommend the soft-release method to be used in future reintroduction attempts of Antilocapra, since more than $50 \%$ of specimens from hard-releases died because of capture stress, or were dispersed, and thus reduced the reintroduction success chances. Rev. Biol. Trop. 65 (3): 1208-1214. Epub 2017 September 01.
\end{abstract}

Key words: Antilocapra americana mexicana, dispersions, hard release, myopathy, soft release.

El éxito de la reintroducción de fauna silvestre depende de diferentes factores, que incluyen el método de captura, el número de animales a liberar, el tiempo empleado para el transporte y el tiempo antes de la liberación. Entre estos, el tiempo que transcurre antes de la liberación, por ejemplo, el de liberación inmediata comparado con la liberación de adaptación, puede tener un efecto significativo en el éxito de los animales trasladados, especialmente para factores como supervivencia y fidelidad al sitio de liberación (Nielson, 1988). Actualmente, se reconocen dos tipos de liberación, la inmediata y con adaptación. La liberación inmediata se define como el transporte de los animales del sitio de captura a su nuevo hábitat, donde son in mediatamente liberados (Nielson, 1988). Por su parte, la liberación con adaptación, se refiere a la liberación de los animales translocados después de un tiempo de aclimatación en un corral con alimento, agua y cobertura ad libitum, sin presión humana (Nielson, 1988).

Para mamíferos existen experiencias anteriores de translocación de otras especies de fauna silvestre, que se han beneficiado con el método de liberación con adaptación, como son el oso negro (Ursus americanus; Eastridge 
\& Clark, 2001), el pecarí (Tayassu tajacu; Porter, 2006), el ciervo colorado (Cervus elaphus; Gilbert, 2007), venados buras (Odoicoileus hemionus; Martínez, 2009) entre otros.

El Antilocapra americana o berrendo fue una especie abundante en las planicies del norte de México, que con la llegada de la minería y ganadería a finales del siglo XIX fue desapareciendo (Leopold, 1959; O’Gara \& Yoakum, 2004 Cancino, 2005). A principios del siglo XX, se reportaron hasta 600 berrendos en Coahuila (Nelson, 1927). Baker (1956), describe un grupo de 50 a 100 individuos en el Valle de la Encantada y el mismo Baker (1958) reportó que el remanente de berrendos de la Encantada, estaba destinado a desaparecer por los cazadores que abastecían de carne a los campos de los mineros. González y Lafón (1993) se refieren a un posible avistamiento de un pequeño grupo de berrendos en las proximidades de Boquillas del Carmen en el año de 1985. En comunicación personal (2009) con el Sr. Juan Villareal de San José de Piedras, Municipio de Ocampo, reportaron un grupo de berrendos entre el rancho La Pantera, Coahuila y el Ejido Las Cruces, Chihuahua, que cruzan de Estados Unidos a México. Los machos pesan de 45-60 kg, mientras que las hembras pesan entre 35 y $45 \mathrm{~kg}$. Presenta una longitud corporal de 1.30 a $1.50 \mathrm{~m}$, una alzada a la cruz de 70 a $80 \mathrm{~cm}$, un largo de cola de $10 \mathrm{~cm}$ y una longitud de oreja de $15 \mathrm{~cm}$. Ambos sexos poseen cuernos negros y perpendiculares, con la punta dirigida hacia adentro, que mudan cada año; en los machos los cuernos son más grandes y están ramificados (125 a $450 \mathrm{~mm})$, mientras que las hembras los tienen cortos y sin ramificar (25 a $150 \mathrm{~mm}$ ). De pelaje hueco y color contrastante de blanco en el vientre y parte interior de las patas, con café-rojizo o café cenizo en la parte superior. También son de color blanco en la grupa, las bandas que tienen en el cuello, los labios, los cachetes y la base de los cuernos. Presentan una crin de color negro. Los machos por lo general tienen la cara más oscura y poseen debajo de las orejas unas manchas que semejan unas "patillas". Los pelos de la grupa son los más largos del cuerpo y los pueden levantar y/o contraer como señal de alarma (Cancino, 2005; Secretaría de Medio Ambiente y Recursos Naturales [SEMARNAT], 2009).

El primer intento de reintroducción del berrendo en México se realizó en 1967, en la Isla Tiburón, Sonora, sin embargo, fracasó en un período menor a tres años (Valdéz \& Manterola, 2006; Secretaría de Medio Ambiente y Recursos Naturales [SEMARNAT], 2009). Una década más tarde, se trasladó un grupo de 22 berrendos provenientes de Colorado, Estados Unidos, a Sonora sin conocer detalles del manejo (Valdéz \& Manterola, 2006). En 1972, se trasladó un grupo de 52 berrendos (19 machos y 33 hembras) de Nuevo Mexico, Estados Unidos al rancho Guadalupe, municipio de Ramos, San Luis Potosí. Sin embargo, la sequía, la dispersión y depredación de crías por coyotes, fueron algunos de los factores de su desaparición. En 1996, se inició la recuperación del berrendo en el estado de Coahuila, en donde se liberó un grupo de 65 individuos en el Valle Colombia, municipio de Ocampo, provenientes de Carrizo, Nuevo México. Durante primer año, algunos individuos y pequeños grupos se dispersaron, y el tamaño de la manada se redujo $54 \%$. Y con el fin de reforzar la primera manada y asegurar la permanencia de la especie en el Valle Colombia en 1998, se realizó una segunda liberación de 85 berrendos. Los primeros años la manada se mantuvo en promedio de 50 a 70 animales, principalmente por efecto de la dispersión, sin embargo, este grupo fue afectado por depredación por coyotes, y el grupo se mantuvo en los últimos cuatro años en un promedio de 35 individuos. Por otro lado, se han hecho algunos traslados de berrendos con el propósito de exhibición, en Caborca, Sonora; Montemorelos, Nuevo León (Valdéz \& Manterola, 2006), y también en Guerrero y Acuña, Coahuila; y Zacatecas, sin éxito. Según la NOM-059-SEMARNAT-2010, esta especie se le considera en peligro de extinción (Secretaría del Medio Ambiente y Recursos Naturales Pesqueros [SEMARNAP], 2010; Convención sobre el Comercio Internacional de Especies Amenazadas de Fauna y Flora Silvestres 
[CITES], 2010; Unión Internacional para la Conservación de la Naturaleza [IUCN], 2010).

En 2006, se inició esfuerzos conjuntos entre la empresa CEMEX (Cementos Mexicanos) y La Secretaría de Medio Ambiente y Recursos Naturales (SEMARNAT) a través de la Comisión Nacional de Áreas Naturales Protegidas (CONANP) con el Programa de Conservación y Aprovechamiento del Berrendo, el Instituto Nacional de Ecología (INE), la Comisión Nacional para el Conocimiento y Uso de la Biodiversidad (CONABIO), la Procuraduría Federal de Protección al Ambiente (PROFEPA), la Dirección General de Vida Silvestre (DGVS), el sector ambiental de Gobiernos Estatales y Estados Unidos, New Mexico Fish and Wildlife Department (NMDGF), de un plan para restaurar las poblaciones del berrendo en las planicies de Maderas del Carmen. Amparados en este proyecto, el objetivo de este estudio fue evaluar el éxito de sobrevivencia y dispersión entre el método de liberación inmediata y la liberación con adaptación, en dos grupos de berrendos trasladados, al reconocer que los principales factores que afectan los programas de reintroducción son la dispersión y la mortalidad por estrés de captura en los primeros días de la liberación.

\section{MATERIALES Y MÉTODOS}

Maderas del Carmen se ubica en el extremo noroeste del estado de Coahuila (en la frontera con Texas, E.U.A.), entre las coordenadas $\left(28^{\circ} 42^{\prime}-29^{\circ} 21^{\prime} \mathrm{N} \& 102^{\circ} 22^{\prime}\right.$ - $102^{\circ} 55^{\prime}$ W). La parte norte y noroeste del área, está limitada por el Río Bravo y, colinda con el Parque Nacional Big Bend; al oeste y al sur por la carretera Melchor Múzquiz-Boquillas del Carmen y al este por el camino de terracería El Melón-La Linda; políticamente forma parte de los municipios de Ocampo, Acuña y Múzquiz. El rango de elevaciones va desde los $500 \mathrm{~m}$ en los márgenes del Río Bravo hasta los $2720 \mathrm{~m}$ en los picos más altos. La temperatura media anual es de $22{ }^{\circ} \mathrm{C}$, con temperaturas en invierno por debajo de los $0{ }^{\circ} \mathrm{C}$. La precipitación en verano que varía de 100-200 mm en las colinas bajas hasta 200-300 mm en las partes más altas. Las comunidades vegetales son Matorral Desértico Chihuahuense (micrófilo, rosetófilo, halófilo y gypsófilo), Matorral Submontano, Zacatal y Bosque de Montaña (pino, encino y oyamel) de acuerdo al gradiente altitudinal (Secretaría de Medio Ambiente y Recursos Naturales y Pesqueros [SEMARNAP], 1997).

En 2006 se inició el programa de reintroducción del berrendo en un predio de 3000 hectáreas de matorral desértico micrófilo secundario rehabilitado con rodillo aereador y se instaló una red de nueve bebederos a $1.5 \mathrm{~km}$ de distancia uno de otro.

El tres de marzo 2009 con la técnica de trampa corral (Fisher, 1942) fueron capturados 45 (23 machos y 22 hembras) berrendos mayores de tres años en Nara Visa, Nuevo México E.U.A., y fueron marcados con arete para su identificación posterior. Para esto, un helicóptero une y arrea al grupo de berrendos a la trampa corral. La trampa corral está diseñada por dos cercas en forma de "V" donde la parte más ancha es la entrada a la trampa, y en la parte final y más angosta, está el corral de manejo y embarque, para después ser transportados hasta el valle oeste de Maderas del Carmen, un día después de la captura. Al arribar al punto de liberación, se les dejó en reposo por un lapso de 14 horas en los remolques en donde se les transportó, antes de proceder a su liberación.

El segundo grupo de 55 adultos (5 machos y 50 hembras) fueron capturados el ocho de marzo del 2010 en Carrizos, Nuevo México E.U.A., se marcaron con aretes de diferente color y numeración para diferenciarlo del primer grupo, transportaron y se confinaron en un corral de pre-liberación. Con este fin, se cercaron cuatro hectáreas con malla venadera de $2.4 \mathrm{~m}$ de alto con $70 \mathrm{~cm}$ de malla como piso al exterior de ella, para restringir la entrada de depredadores; se diseñó con esquinas redondeadas, para evitar estrellamientos dentro del corral, también se instalaron dos bebederos, y se suplementó a los berrendos con $80 \mathrm{~kg}$ de alfalfa cada segundo día, durante 17 días hasta su liberación definitiva. El seguimiento se realizó con la técnica de conteo terrestre (Wooley 
\& Lindzey, 1994; Miranda, 2000) para ambos grupos, se hizo recorridos diarios, en cuatrimoto a lo largo de $20 \mathrm{~km}$ a una velocidad de 5 $\mathrm{km} / \mathrm{h}$, durante las siguientes tres semanas después de las liberaciones en marzo 2009 y marzo 2010; los recorridos se realizaron de 7 a 11 de la mañana, y se procuró localizar a los animales mediante observación directa, con binoculares $10 \mathrm{x} 40 \mathrm{y}$ un telescopio $30 \mathrm{x}$, y se registró su ubicación, tamaño y estructura del grupo; también se anotó mortalidades y posibles causas muerte. Los resultados obtenidos se analizaron mediante una $\chi^{2}$.

\section{RESULTADOS}

Con el método de liberación inmediata de los berrendos en las planicies de Maderas del Carmen, el $31 \%(n=13,5$ hembras y 8 machos) logró su establecimiento, el $46 \%$ ( $\mathrm{n}=$ 21, 9 hembras y 12 machos) se dispersó, y el $24 \%$ ( $n=11,8$ hembras y 3 machos) murieron en la primer semana después de la liberación. La mortalidad fue causada fue la afectación por miopatía, dadas las características de atrofia muscular encontrada en las necropsias en campo. Durante la primera semana, se registraron individuos solitarios o en grupos no mayores de tres en el extremo norte del área de estudio, se les observó alimentándose de Opuntia spp., Viguiera stenoloba y Croton lechleri entre otras.

Por su parte, con el método de liberación con adaptación practicado en la misma localidad, los resultados fueron mejores: el $83 \%(n=$ 46, 43 hembras y 3 machos) se estableció, el $4 \%(\mathrm{n}=2,2$ hembras) murió por estrés en el corral de adaptación, y el $13 \%(n=7,5$ hembras y 2 machos) migró hacia el norte del punto liberación sin registrar regreso. Mientras este segundo grupo se encontraba en el corral de adaptación, se observó que el grupo fundador visitó el corral en diferentes ocasiones. Esta interacción con el grupo del corral parece haber sido muy útil al momento de la liberación, pues el grupo fundador sirvió como ancla al grupo recién liberado.

En la figura 1 se muestra la comparación entre ambos métodos de liberación. Con el análisis de la prueba de chi cuadrado $\left(\chi^{2}=\right.$ 30.998, g.1. $=2, p<0.0001)$; encontramos un resultado altamente significativo entre los dos métodos de liberación, siendo la liberación con adaptación el de mejor resultado, al obtener

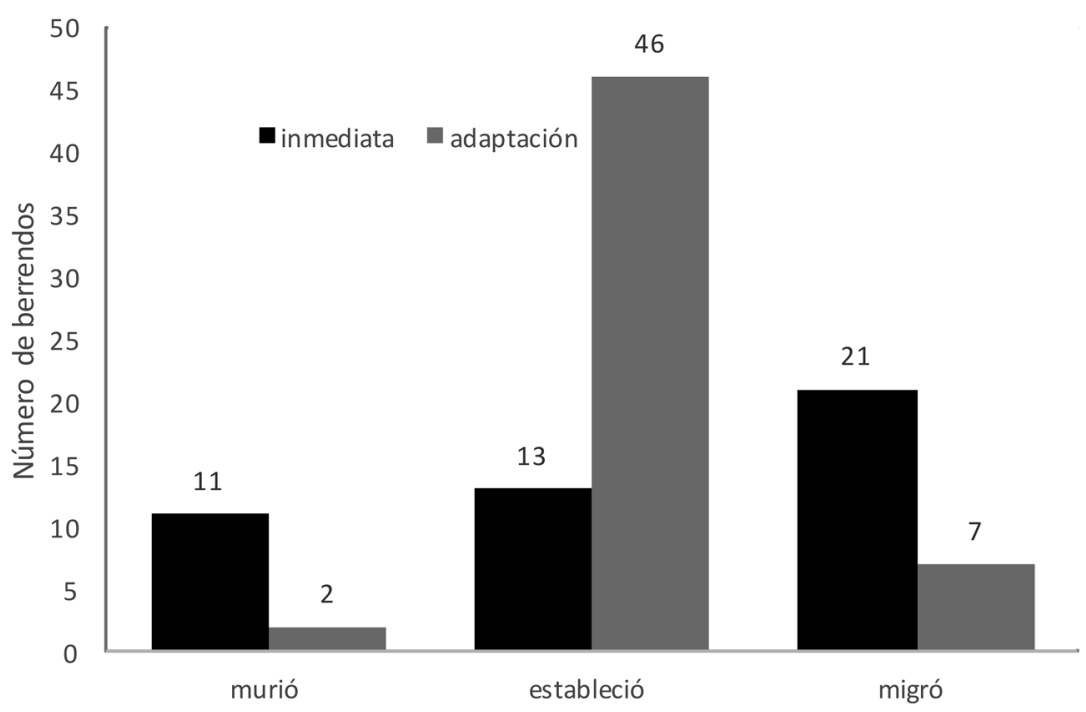

Fig. 1. Liberación inmediata versus comparación con adaptación de Antilocapra americana en Maderas del Carmen, Coahuila, México. 
mejores tasas de supervivencia y fidelidad al sitio de liberación.

\section{DISCUSIÓN}

El método de liberación con adaptación empleado en las planicies de Maderas del Carmen, Coahuila, demostró su eficiencia al comparar sus resultados con los obtenidos con el método de liberación inmediata. Resultados similares reportan Fatooh, Russi y Goldsmith (1994) quienes mencionan que en los primeros días de una liberación inmediata, registraron una dispersión del $67 \%$ (29 animales) de un grupo de 43 berrendos, estableciéndose un total de seis individuos (13\%). En una segunda liberación, Fatooh et al. (1994) evaluó la liberación de 24 berrendos al utilizar un corral establecido de cinco hectáreas provisto de agua y forra$\mathrm{je}$, con una cerca de $1.3 \mathrm{~m}$ de alto, en donde mantuvieron a los animales por ocho días, en esta liberación con el corral de adaptación, los animales permanecieron como un solo grupo, cinco días después se encontraron con el grupo fundador de seis berrendos, y refiere que en las liberaciones posteriores los berrendos fueron beneficiados por la asociación con los primeros berrendos liberados, los cuales ya tenían experiencia en el terreno. En otro estudio, Valdez y Manterola (2006), reportaron que en el Valle Colombia, Coahuila después de la liberación inmediata, el $54 \%$ se estableció, y por ende $46 \%$ migró, y en comunicación personal Valdez (2009) comentó que la mitad murió por estrés de captura.

Por otro lado, Koch y Yoakum (2002) reportaron para una reintroducción de berrendo en Carrizos, California E.U.A., que después de la captura, los trasladaron inmediatamente en tráiler aproximadamente $966 \mathrm{~km}$ hasta el punto en donde fueron liberados, en la liberación inmediata los animales se estresaron mucho y no se reagruparon. Un año después en la siguiente liberación realizada por los mismos autores, mantuvieron al grupo en un corral por 24 horas (liberación con adaptación), y reportaron que este grupo se mantuvo calmado y en grupo después de la liberación; sin embargo, no aportaron información de mortalidad por miopatía o dispersión.

La liberación con adaptación para esta especie dio resultados altamente significati$\operatorname{vos}\left(\chi^{2}=2, \alpha=0.05, p=0.0001\right)$ y mejores, al compararlos con los de liberación inmediata. $\mathrm{Al}$ dejar los berrendos en confinamiento en el corral con alimento, agua, cobertura y espacio, estos se mantuvieron calmados, y en un solo grupo al momento de ser liberados; por lo tanto, el método de liberación con adaptación, se recomienda utilizarlo en futuras liberaciones de berrendo, pues más del $50 \%$ de los berrendos procedentes de liberaciones inmediatas, murieron por estrés de captura o se dispersaron y con ello, se redujeron las posibilidades del éxito del traslado.

Posterior a la liberación y en comunicación personal con Javier Ochoa (biólogo de CONANP) nos reportó haber observado berrendos marzo 2009 en los ejidos de Jaboncillos (2 hembras y un macho) a $36 \mathrm{~km} \mathrm{al}$ norte del punto de su liberación; también en, la Unión (dos hembras) a $44 \mathrm{~km}$ al noroeste, así como varios avistamientos de berrendos por los pobladores en la carretera a Boquillas del Carmen, en el tramo de San Miguel y Las Norias y en junio 2009, encontraron un berrendo atropellado en la cuesta Malena.

Este proyecto también permitió el análisis de otros aspectos vinculados con el traslado de organismos. Todo traslado, no solo del berrendo, debe contar con un plan de manejo, suplemento alimenticio y un monitoreo de la especie después de su liberación. También, se estima importante equipar a los individuos con radio transmisores de telemetría y/o GPS, para optimizar el monitoreo de desplazamiento, valorar o determinar las causas de muerte e identificar a las hembras productivas.

El futuro del berrendo en el estado de Coahuila va a depender de nuevas liberaciones y del establecimiento de programas de educación y difusión acerca de la especie, así como ampliar la investigación, monitoreo y manejo de hábitat a largo plazo. También, el éxito se dará a medida que se involucre a los propietarios de predios de la región, en donde 
habita la especie y se sigan haciendo esfuerzos coordinados entre dependencias de gobierno, iniciativa privada, sectores académicos, $\mathrm{ONG}$ de conservación y público en general.

\section{AGRADECIMIENTOS}

Agradecemos a CEMEX por el financiamiento del estudio, con el cual obtuve el grado de Master en Ciencias, Manejo de Vida Silvestre y Desarrollo Sustentable en la Facultad de Ciencias Biológicas de la Universidad Autónoma Nuevo León (H.S.G.), también agradecemos a Billy Pat McKinney y personal del proyecto El Carmen-CEMEX por esta iniciativa de conservación y al personal del Laboratorio de Ornitología de U. A. N. L. por el apoyo brindando.

\section{RESUMEN}

La reintroducción de especies desaparecidas de mamíferos en sus hábitats históricos ha sido recientemente de gran interés. Desde 1967 se han realizado varios intentos de reintroducción del berrendo en México, ninguno ha sido exitoso. El objetivo de este estudio es proveer información que contribuya a evaluar el éxito de la liberación. El estudio se sustentó en el monitoreo de 100 individuos (70 hembras y 25 machos) capturados en Nuevo México E.U.A. y liberados en Maderas del Carmen, Coahuila, en dos etapas, el primer grupo de 45 individuos (20 hembras, 25 machos) liberado en la vertiente occidental de Maderas del Carmen en marzo 2009 y el segundo grupo en marzo 2010 con 55 individuos más (50 hembra, 5 machos). Se comparó la dispersión y mortalidad entre el método de liberación de adaptación vs liberación inmediata. La liberación con adaptación para esta especie dio un resultado altamente significativo $\left(\chi^{2}=2, \alpha=0.05, p=0.0001\right)$. Los resultados de los métodos de liberación inmediata fueron de $23 \%$ de mortalidad y $46 \%$ de dispersión y los de la liberación de adaptación fueron de 4 y $13 \%$ respectivamente. El método de liberación de adaptación es más benévolo que la liberación inmediata ayudando a reducir la dispersión y mortalidad por miopatía en translocaciones de berrendo. Por lo que el método de liberación con adaptación se recomienda utilizarlo en futuras liberaciones de berrendo ya que demuestra que más del $50 \%$ de los berrendos procedentes de liberaciones inmediatas mueren por estrés de captura o se dispersan y con ello, se reducen las posibilidades de éxito.

Palabras claves: Antilocapra americana mexica$n a$, dispersión, liberación inmediata, liberación de adaptación, miopatía.

\section{REFERENCIAS}

Baker, R. H. (1956). Mammals of Coahuila Mexico. Kansas, E.U.A.: Museum of Natural History.

Baker, R. H. (1958). The future of wildlife in northern Mexico a problem in conservation education. Transactions of the 23rd North American Wildlife Conference, $23,567-575$.

Cancino, J. (2005). Familia Antilocapridae, Berrendo. En G. Ceballos \& O. Giselle (Eds.), Los Mamíferos silvestres de México (pp. 502-504). México, D.F., México: Comisión Nacional para el Conocimiento y Uso de la Biodiversidad.

Convención sobre el Comercio Internacional de Especies Amenazadas de Fauna y Flora Silvestres. (2010). Apéndices. Recuperado de https://cites.org/esp/app/ index.php

Eastridge, R., \& Clark, J. D. (2001). Evaluation of 2 soft release techniques to reintroduce black bears. Wildlife Society Bulletin, 29(4), 1163.

Fatooh, J., Russi, T., \& Goldsmith A. (1994). Pronghorn reintroduction to Mono County, California: 12 years after. Pronghorn Antelope Workshop, 16, 35-49.

Fisher, L. W. (1942). Live tramping Texas antelopes. Journal Wildlife Management, 6, 231-236.

Gibert, S. (2007). Dinámica poblacional y preferencia de hábitat de un grupo de wapitíes (Cervus elaphus) reintroducido en la Sierra Maderas del Carmen (Tesis de Maestría). U.A.N.L., Linares, México.

González, A. \& Lafón, A. (1993). Distribución y estado actual del berrendo (Antilocapra americana) en México. En R. A. Medellín \& G. Ceballos (Eds.), Avances en el estudio de los mamíferos de México (pp. 409-420). México D.F., México: Asociación Mexicana de Mastología, A.C.

Unión Internacional para la Conservación de la Naturaleza (2010). Red List of threatened species. Recuperado de www.iucnredlist.org

Koch, A., \& Yoakum, J. (2002). Reintroduction and status of pronghorn on the Carrizo Plain National Monument and surrounding areas in southern. Pronghorn Antelope Workshop, 20, 25-41.

Leopold, A. (1959). Fauna Silvestre de México Aves y Mamíferos de Caza. México D. F., México: Instituto Mexicano de Recursos Renovables.

Martínez, L. (2009). Site fidelity and post release movements of translocated mule deer in northern Coahuila, Mexico (Tesis de Maestría). Sul Ross State University, Alpine. E.U.A.

Miranda, E. P. (2000). Monitoreo de una población de berrendo (Antilocapra americana mexicana) 
reintroducida el noroeste de Coahuila. (Tesis Maestría). U.A.N.L. Linares, México.

Nelson, E. W. (1927). Status of Pronghorned antilope. 1922-1924. U.S. Departament Agriculture Bulletin, 1346, 1-66.

Nielson, L. (1988). Definitions, considerations, and guidelines for translocation of wild animals, En L. Nielsen \& R. D. Brown (Eds.), Translocation of wild animals (pp 12-52). Kingsville, E.U.A.: Caesar and Kleberg Wildlife Research Institute.

Secretaría de Medio Ambiente y Recursos Naturales y Pesqueros [SEMARNAP]. (1997). Programa de Manejo del Área de Protección de Flora y Fauna Maderas del Carmen, Coahuila, México. México D.F., México: Instituto Nacional de Ecología.

Secretaría del Medio Ambiente y Recursos Naturales Pesqueros [SEMARNAP]. (2010). Norma Oficial Mexicana NOM-059-SEMARNAT-2010 Diario Oficial de la Federación. Recuperado de http://www. profepa.gob.mx/innovaportal/file/435/1/NOM_059 SEMARNAT_2010.pdf
Secretaría de Medio Ambiente y Recursos Terrestres [SEMARNAT]. (2009). Programa de acción para la conservación de la especie: Berrendo (Antilocapra americana). México D.F., México: Comisión Nacional de Áreas Naturales Protegidas.

O’Gara, B., \& Yoakum, J. (2004). Pronghorn Ecology and Management. Boulder, E.U.A.: University Press of Colorado.

Porter, B. (2006). Evaluation of collared peccary translocations in the Texas Hill Country (Tesis Maestría). Texas A\&M University, Kingsville, E.U.A.

Valdéz, M. \& Manterola, C. (2006). Reintroducción del berrendo en Coahuila. En M. Valdéz, E. Peters, \& E. Palledares (Eds.), El berrendo en México acciones de conservación (pp. 97-111). México, D. F., México: Agrupación Sierra Madre.

Woolley, T. P., \& Lindzey, F. G. (1994). Relative precision and sources of bias in pronghorn sex and age composition surveys. Journal Wildlife Management, 61(1), 57-63. 